\title{
Radio Emission from Binary Stars in the AB Doradus Moving Group
}

\author{
R. Azulay ${ }^{1}$, J.C. Guirado ${ }^{1,2}$, J.M. Marcaide ${ }^{1}$, I. Martí-Vidal ${ }^{3}$, and \\ E. $\operatorname{Ros}^{4,1,2}$ \\ ${ }^{1}$ Departament d'Astronomia i Astrofísica, Universitat de València, E-46100 Burjassot, \\ València, Spain \\ email: rebecca.azulay@uv.es \\ ${ }^{2}$ Observatori Astronòmic, Universitat de València, E-46980 Paterna, València, Spain \\ ${ }^{3}$ Onsala Space Observatory, Chalmers University of Technology, SE-439 92, Onsala, Sweden \\ ${ }^{4}$ Max-Planck-Institut für Radioastronomie, D-53121 Bonn, Germany
}

\begin{abstract}
Precise determination of dynamical masses of pre-main-sequence stars is essential for calibrating stellar evolution models, that are widely used to derive theoretical masses of young low-mass objects. We have determined the individual masses of the pair $\mathrm{AB} \mathrm{Dor} \mathrm{Ba} / \mathrm{Bb}$ using Australian Long Baseline Array observations and archive infrared data, as part of a larger program directed to monitor binary systems in the AB Doradus moving group. We have detected, for the first time, compact radio emission from both stars. This has allowed us to determine the orbital parameters of both the relative and absolute orbits and, consequently, their individual dynamical masses: $0.28 \pm 0.05 \mathrm{M}_{\odot}$ and $0.25 \pm 0.05 \mathrm{M}_{\odot}$. Comparisons of the dynamical masses with the prediction of pre-main-sequence (PMS) evolutionary models show that the models underpredict the dynamical masses of the binary components $\mathrm{Ba}$ and $\mathrm{Bb}$ by $10-30 \%$ and $10-40 \%$, respectively.
\end{abstract}

Keywords. astrometry, binaries (including multiple): close, stars: fundamental parameters, stars: pre-main sequence

\section{Introduction}

An improvement of the calibration of PMS evolutionary tracks should necessarily come from the precise determination of dynamical masses of PMS stars. Binary stars in young, nearby moving groups are good candidates. We selected the AB Doradus moving group (AB Dor-MG) as the best-suited association to apply radio-based high-precision astrometric techniques to study binary systems. This choice is well supported by the systems mean distance to the Sun (30 pc), its reasonably well known age (50-70 Myr; Janson et al. 2007; Guirado et al. 2011), and the presence of radio emission in some of its active members (Guirado et al. 2006; Azulay et al. 2014, 2015). Following the list of AB Dor-MG members in Torres et al. (2008), we initiated a VLA/VLBI program to monitor binary systems known to host low-mass companions, and which are likely to present radio emission.

\section{Long Baseline Array observations of AB Dor B}

$\mathrm{AB}$ Dor B is a close binary which consists of two components, AB Dor Ba and AB Dor Bb, separated by $\sim 60$ mas (Guirado et al. 2006; Janson et al. 2007). We observed this star between 2007 and 2013 with the Australian Long Baseline Array at $8.4 \mathrm{GHz}$ (Azulay et al. 2015). With these observations, we confirmed that both components are compact and strong radio emitters. We could determine the relative position of one component 


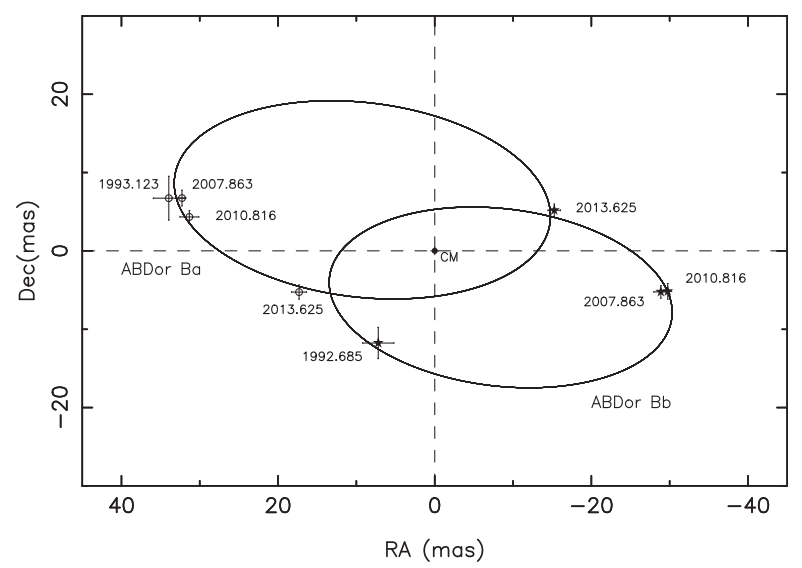

Figure 1. Absolute orbits of AB Dor Ba and AB Dor Bb. The positions of components Ba (circles) and $\mathrm{Bb}$ (star symbols) are marked along with their respective epochs. The center of mass $(\mathrm{CM})$ of the system is placed at the origin.

with respect to the other and the absolute position of each component with respect to an external quasar. We also included NIR data available in the literature (Wolter et al. 2014, and references therein) to determine simultaneously both the relative and the individual orbits of each pair (Fig. 1). The best orbit corresponds to a period of $0.986 \pm 0.008 \mathrm{yr}$. The masses of the components are $0.28 \pm 0.05 \mathrm{M}_{\odot}$ and $0.25 \pm 0.05 \mathrm{M}_{\odot}$ for $\mathrm{AB} \mathrm{Dor} \mathrm{Ba}$ and $\mathrm{AB}$ Dor $\mathrm{Bb}$, respectively.

\section{Conclusions}

Comparison of the dynamical masses with the prediction of PMS models show that the models underpredict the dynamical masses of the binary components $\mathrm{Ba}$ and $\mathrm{Bb}$ by 10-30\% and 10-40\%, respectively, underlining the known tendency of these models to underpredict the masses (Hillenbrand \& White 2004; Mathieu et al. 2007), yet within $2-\sigma$ of the predicted values. Some of the stellar models considered favour an age between 50 and $100 \mathrm{Myr}$ for this system, meanwhile others predict older ages. Our ongoing VLBI program includes European VLBI Network $5 \mathrm{GHz}$ observations of other stars belonging to the AB Dor-MG such as EK Draconis, LO Pegasus, PW Andromedae, and HD 160934. In particular, for the latter star we have determined a period of $10.33 \pm 0.06 \mathrm{yr}$ and preliminary values of the individual masses of $\sim 0.7 \mathrm{M}_{\odot}$ for the component $\mathrm{A}$ and $\sim 0.5 \mathrm{M}_{\odot}$ for the component c (Azulay et al., in preparation).

\section{References}

Azulay, R., Guirado, J. C., Marcaide, J. M., et al. 2014, A\&A, 561, A38

Azulay, R., Guirado, J. C., Marcaide, J. M., et al. 2015, A\&A, 578, A16

Guirado, J. C., Martí-Vidal, I., Marcaide, J. M., et al. 2006, A\&A, 446, 733

Guirado, J. C., Marcaide, J. M., Martí-Vidal, I., et al. 2011, Aछ A, 533, A106

Hillenbrand, L. A. \& White, R. J. 2004, ApJ, 604, 741

Janson, M., Brandner, W., Lenzen, R., et al. 2007, A $\& A$, 462, 615

Mathieu, R. D., Baraffe, I., Simon, M., et al. 2007, Protostars and Planets V, 411

Torres, C. A. O., Quast, G. R., Melo, C. H. F., et al. 2008, Handbook of Star Forming Regions, Volume II, 757

Wolter, U., Czesla, S., Fuhrmeister, B., et al. 2014, A\&GA, 570, AA95 